\title{
Medida de la satisfacción estudiantil a partir de la satisfacción con la vida: una relación de componentes ${ }^{1}$
}

\author{
Alfonso Ríos Sánchez ${ }^{2}$ \\ I.E. Bajo Cañada ${ }^{3}$ \\ Universidad de Caldas, Colombia ${ }^{4}$ \\ alriosan@hotmail.com
}

1 Artículo de investigación

2 Magister en Innovación e Investigación en Educación. Universidad Nacional de Educación a Distancia, UNED, España

3 Docente

4 Tutor catedrático 


\title{
Medida de la satisfacción estudiantil a partir de la satisfacción con la vida: una relación de componentes
}

\section{Resumen}

El estudio buscó conocer variables significativas de la satisfacción respecto a la calidad de la educación, vinculadas a la satisfacción con la vida. Se llevó a cabo durante el curso 2012-2013, con una población de 235 estudiantes de 3. y 4. de la Educación Secundaria Obligatoria (ESO). Se configuró un instrumento de medida basado en las subdimensiones del modelo de evaluación integral de Scheerens y de programas de Stufflebeam. Se analizó la relación de las subdimensiones por medio de un análisis de regresión. Las variables empatía, logros personales y reconocimiento del éxito, fueron las más significativas como predictoras de la satisfacción con la calidad de la educación, en vinculación con la satisfacción con la vida. Estas variables pueden ayudarnos a superar una visión reducida de la satisfacción, enfatizando áreas de la gestión del centro que pueden ser fortalecidas y que los estudiantes estiman relevantes para su desarrollo educativo.

Palabras clave: satisfacción estudiantil, satisfacción con la vida, bienestar subjetivo, calidad de la educación, gestión de centro, análisis de regresión lineal.

\section{Measuring student satisfaction from their satisfaction in life: a relationship of different components.}

\begin{abstract}
The study sought to find significant satisfaction variables regarding the quality of education concomitantly linked to life satisfaction. It was conducted during 2012-2013, with a population of 235 students of 3rd and 4th graders from Secondary School Education. A measuring instrument based on the sub-dimensional model of comprehensive evaluation and program Scheerens Stufflebeam was created. The ratio of the sub dimensions through regression analysis was studied. Empathy, personal achievements and recognition of success, were the most significant variables as predictors of satisfaction with the quality of education in connection with satisfaction with life. These variables can help us overcome a reduced view on satisfaction, emphasizing areas of school management that can be strengthened and which students consider relevant to their educational development.
\end{abstract}

Keywords: student satisfaction, life satisfaction, subjective well-being, quality of education, center management, linear regression analysis.

\section{Medida da satisfação dos estudantes a partir da satisfação com a vida: uma relação de componentes}

\section{Resumo}

O estudo procurou encontrar variáveis significativas de satisfação com a qualidade da educação ligadas à satisfação com a vida. Foi realizado durante o curso 2012-2013, com uma população de 235 estudantes de 3ㅇ e 4응 da Educação Secundária Obrigatória (ESO). Criou-se uma ferramenta de medição baseada nas sub-dimensões do modelo de avaliação integral de Scheerens e de programas de Stufflebeam. Analisou-se a relação das subdimensões através de uma análise da regressão. As variáveis Empatia, Sucessos pessoais e Reconhecimento do sucesso, foram as mais significativas como vaticinadoras da satisfação com a qualidade da educação, em relação à satisfação com a vida. Estas variáveis podem nos ajudar na superação de uma perspectiva reduzida da satisfação, enfatizando aspectos da administração do centro que podem ser fortalecidos e que os estudantes consideram relevantes para o seu desenvolvimento educativo.

Palavras chave: esatisfação estudantil, satisfação com a vida, bem-estar subjetivo, qualidade da educação, administração do centro, análise da regressão linear. 


\section{Introducción}

El propósito básico de la educación secundaria es brindar a los jóvenes la formación necesaria que les habilite para el desarrollo de sus capacidades y destrezas a lo largo de la vida (Gardner, 1993; 1999; Morin, 2002a; Pérez, 2005). La educación integral busca unir todos los aspectos que hacen parte del proceso educativo, estableciendo conexiones entre las diferentes asignaturas y las diversas experiencias que vive el estudiante, habilitándolo para adaptarse a las nuevas situaciones y combinar los conocimientos de forma inteligente.

La oportuna relación entre los diferentes elementos que forman parte de los programas educativos es constatada por medio de la evaluación institucional. Los resultados de esta se usarán para mejorar y adecuar las condiciones necesarias, el material y procedimientos adecuados, a fin de alcanzar las metas propuestas, que se harán evidentes en el producto. Desde hace varios años se realizan investigaciones que buscan conocer las características requeridas por el centro educativo para el buen desarrollo pedagógico (Seligman \& Csikszentmihalyi, 2000). Y uno de los ámbitos de estos estudio es el bienestar subjetivo considerado fundamento indispensable para la educación integral. Muchas instituciones educativas realizan evaluaciones de sus procedimientos, empleando, entre otras, encuestas de satisfacción que buscan conocer el grado de bienestar que obtienen sus estudiantes con la educación que reciben. Las investigaciones sobre la satisfacción escolar se han centrado principalmente en las consecuencias de la insatisfacción con la escuela: la deserción y el abandono (McCullough, Huebner, \& Laughlin, 2000). El disfrute y el bienestar subjetivo, forman parte de los indicadores del desarrollo personal, y estos pueden ser medidos en los centros educativos por medio de la satisfacción. Son pocos los estudios que indagan sobre la satisfacción empleando indicadores basados en el bienestar subjetivo en la escuela secundaria. La indagación de la satisfacción educativa de indicadores basados en el bienestar subjetivo, puede ser una manera de comprender la repercusión que tiene la formación brindada por el centro en el desarrollo y la estructuración de la personalidad de los jóvenes estudiantes.

La satisfacción educativa puede estar muy relacionada con la satisfacción general con la vida (Huebner, 1994), (Diener 1994). La identificación de las variables más significativas del proceso educativo tomando como referente las condiciones socioemocionales, puede brindar a los centros educativos la información necesaria sobre que tanto está afectando la educación que reciben los estudiantes, y como está afecta el conjunto de la vida. Además, el disfrute puede ser tomado en cuenta como otro indicador personal que forma parte de la satisfacción (Elmore \& Huebner, 2010).

\section{Formulaciones para medir la satisfacción estudiantil}

Las orientaciones teóricas para definir satisfacción están dotadas de variedad de tendencias y corrientes. Algunas la consideran como una respuesta en conformidad 
con expectativas creadas ante un servicio, y otras la consideran como un producto. Se considera que la primera orientación tiene más relación desde una perspectiva formativa. Una definición enmarcada en perspectiva educativa puede partir de la relación que tiene la satisfacción como indicador de calidad, relación que nos da pautas para la conceptualización. Una opción para la definición que se relaciona con los intereses de este estudio, se puede enmarcar en la propuesta que hace Galán (2004) sugiriendo que para definir la calidad de la educación hemos de partir desde una actitud pragmática, basada en la consideración de un conjunto de criterios, los cuales pueden ser evaluados convenientemente.

Sobre dicha opción conceptual operativa, se ha de considerar que son los centros educativos los que eligen los criterios para valorar la calidad de sus procesos, en una relación social con las administraciones educativas, las entidades del sector, y también la normativa vigente. La elección de criterios que hacen los centros educativos, para medir la calidad de sus actividades pedagógicas, pueden contener indicadores que den razón de la eficaz respuesta ante las necesidades formativas y emocionales de los estudiantes.

Siguiendo la definición propuesta por Pérez, Galán, y Quintanal (2012), para que la educación pueda darse, esta se debe llevar a cabo en un contexto específico. El acto educativo es contextualizado, se desarrolla en el ambiente escolar del cual también participan el ambiente social y cultural. Por esta razón, la contextualización de la educación es un factor importante a tener en cuenta, si se desean ampliar los criterios de evaluación sobre la eficacia de los procedimientos, en tanto que nos ayuda a delimitar el modo como puede influir o determinar el contexto. La evaluación de la calidad de la educación, puede establecerse en la consideración relacional de los diferentes componentes y elementos que la constituyen. En este sentido, Gento (2000) nos propone una concepción holística sistemática para evaluar la calidad de la educación, considerando esta evaluación como la conjugación de diversos elementos que involucran el proceso educativo y su interacción con el contexto social.

\section{Medir la satisfacción como relación de componentes}

El modelo integrado de Scheerens (1992), citado por Galán (2004), como también el modelo CIPP de Stufflebeam, citado por Pérez (2006), estructurados por componentes son áreas significativas de gestión para la evaluación del centro educativo. Estos modelos relacionales están estructurados por cuatro componentes: INPUTS, O aspectos generales de entrada cuyos elementos tienen que ver con los suministros efectivos y operativos del servicio educativo; procesos, que corresponde a todas aquellas acciones orgánicamente diseñadas y ejecutadas con objetivos definidos y metas evaluables; producto, el cual contiene todos aquellos resultados esperados y proyectados del acto educativo; y contexto, espacio y ambiente donde se desarrolla el acto educativo. 
El estudio de la eficacia de la calidad de la educación se puede lograr por medio de la vinculación de los diferentes componentes, y dicha vinculación puede hacerse a través de la indagación de los niveles de satisfacción. Este tipo de orientación es basada en los modelos integrados, como el propuesto por Scheerens (1992) y Stufflebeam (1987). El desarrollo de las investigaciones basadas en la relación de componentes, se nos presenta como un paradigma de investigación, que ha pasado de los estudios de correlación, hasta llegar a estudios de modelos causales. Estas técnicas son sustentadas en modelos correlaciónales como los propuestos por Centra y Potter (1989), citado en Galán (2004). El mayor aporte de estos modelos de evaluación, consiste en el establecimiento a priori del tipo y dirección de las relaciones esperadas entre las entradas, procesos y el producto, gracias a que se permitirá un mejor entendimiento del modelo teórico y de las interacciones y los efectos particulares de las variables de entrada y procesos sobre el producto. Investigar la relación de los componentes que forman parte de la educación, comprendiendo su naturaleza y sus condiciones, puede ayudarnos a concentrar la atención sobre cómo los centros educativos están respondiendo ante las necesidades emocionales de los estudiantes.

\section{El contexto educativo, fundamento de la satisfacción estudiantil}

Consideramos que el contexto educativo va más allá de las formulaciones logísticoadministrativas que hacen parte de la gestión del centro. Tengamos en cuenta que el centro está integrado dentro de una circunscripción social y cultural que puede determinar y condicionar gran parte de las actuaciones educativas, pertenencia que nos hace considerar la necesidad de ampliar el componente contexto hacia otros escenarios donde también participan los estudiantes. En este sentido, se ha apostado por una ampliación de la frontera del contexto educativo incluyendo dos elementos. En esta ampliación se ha puesto la línea limítrofe en la capacidad de gestión que tiene el centro sobre el control de los escenarios escolares, es decir, el centro gestiona los escenarios contextuales educativos, pero a su vez este puede ser influenciado por otros escenarios que también hacen parte del contexto.

Basado en el modelo integrado de centro de Scheerens (1989) y de programas de Stufflebeam (1987), se propone un esquema donde se incluyen en el contexto educativo, el contexto familiar y social para la evaluación de calidad de la educación por medio de la satisfacción. 


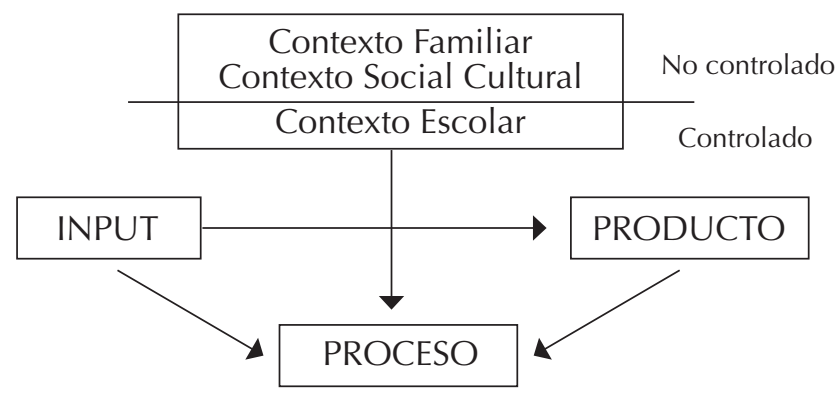

Gráfico 1. Modelo de evaluación del centro.

\section{Satisfacción, una medida percibida y subjetiva}

La satisfacción con la educación puede entenderse como la valoración subjetiva y cognitiva de la calidad percibida de la experiencia vivida en el centro educativo (Baker, Dilly, Aupperlee, \& Patil, 2003). Y su formulación está relacionada con la satisfacción general con la vida. Un espacio para la definición de la satisfacción con la educación es la satisfacción con la vida en general, al proporcionar una evaluación personal vinculando todas las áreas importantes de la vida. Aunque existen variaciones entre los dominios influyentes, esta relación es necesaria para comprender sus características e influencias (Huebner, Suldo, \& Valois, 2003; Huebner, Valois, Paxton, y Drane, 2005; Antaramian, Huebner, \& Valois, 2008). Huebner ha realizado varios estudios sobre la satisfacción educativa, basados en el bienestar subjetivo, dichos estudios buscan fundamentar las consideraciones sobre la evaluación de la calidad educativa como la respuesta que el centro educativo realiza sobre las necesidades emocionales. La teoría del apego (Bowlby 1973), citado por Huebner (2004) es una propuesta conveniente para comprender el establecimiento de la base de las relaciones emocionales fundamentadas desde la edad temprana y para futuras relaciones. Basado en esta teoría Huebner (2004) propone que el centro educativo puede convertirse en un escenario compensatorio y/o complementario para el fortalecimiento socioemocional. De forma similar Diener (1994), ha realizado estudios basados en el bienestar subjetivo dentro de la formación primaria y secundaria, encontrando en estos un amplio campo de acción para comprender mejor las dinámicas de los procesos educativos.

\section{Medir la satisfacción con la escuela a partir de la satisfacción con la vida}

La satisfacción con la escuela, como se ha mencionado con anterioridad, es posible relacionarla con la satisfacción con la vida. Los primeros estudios que realizan análisis de la relación entre la satisfacción con la vida y su repercusión en las diferentes dimensiones de la vida fueron iniciados por Epstein y McParthland (1976). A partir de estos estudios, se considera que el bienestar subjetivo es un fundamento básico 
para la valoración de la satisfacción. Un desarrollo educativo positivo puede ser comprendido por medio de índices óptimos de satisfacción escolar. La medida de este oportuno desarrollo educativo, basado en criterios de eficiencia y eficacia, puede ayudar al fortalecimiento constante del desarrollo curricular del centro. Existen diversos estudios que siguen esta línea de relación en la investigación. Algunos de ellos centran la orientación en variables personales o subjetivas, como determinantes para la satisfacción:

a). Las relaciones afectivas entre estudiantes han sido estudiadas para conocer su influencia en los niveles de satisfacción con la escuela. La investigación que examina las relaciones entre pares y la satisfacción con la vida, sugiere que las relaciones entre iguales son un factor débil para la satisfacción de los estudiantes con la escuela (Huebner, 1991b). Los estudiantes de las escuelas primarias poco relacionan la satisfacción con la escuela, basándose en criterios de satisfacción de su grupo de amigos, esta razón puede estar sustentada en que los estudiantes basan la valoración con la escuela a partir de los criterios que se aprenden en la familia.

b). Se ha examinado la influencia que tiene los factores contextuales del aula escolar sobre la satisfacción de los estudiantes. Baker (1998) examinó la relación entre el clima del aula y la satisfacción con la escuela basado en el estrés escolar. Las conclusiones de este estudio sugieren que el clima escolar tuvo un fuerte efecto directo sobre la satisfacción de los estudiantes con la escuela. El clima escolar puede ser un componente determinante para comprender aspectos de la satisfacción educativa, si es analizado por medio de factores específicos como el estrés escolar. Estos resultados sugieren que para los estudiantes la percepción de un ambiente de clase "cuidado y seguro psicológicamente" es importante para la percepción de los estudiantes sobre sus experiencias escolares (Baker, 1998). Además, sobre el estrés escolar se encontró que tiene un efecto indirecto en la satisfacción de la escuela por influir negativamente en la percepción de la vida familiar, el apoyo social y el autoconcepto académico. Por otra parte, Baker (1999) también estudió la influencia de la calidad de la relación entre profesorestudiante en la satisfacción con la escuela. Entre las conclusiones sugiere que los estudiantes que tenían relaciones más solidarias y de apoyo con los maestros, reportaron mayor satisfacción con la escuela.

c). La relación entre las características personales de los estudiantes (auto concepto/ auto estima) y las condiciones ambientales (clima escolar, estrés escolar, la calidad de la vida familiar, las experiencias negativas de la vida), son estudiadas para comprender su interrelación con la satisfacción educativa (Huebner, 2004). En este estudio se sugiere que el sentido de control interno se asocia con mayores niveles de satisfacción con la escuela, por lo que las variables personales y ambientales contribuyen en los reportes que los estudiantes realizan de la satisfacción con la escuela. 
d). Otros estudios hacen discriminación entre las variables personales y variables ambientales, las relacionan y les dan una orientación específica para comprender la satisfacción educativa basada en satisfacción general con la vida.

Huebner (1991a); McCullough, Huebner \& Laughlin (2000); Baker (1998) y Diener, Suh, Lucas, \& Smith (1999), sugieren que existen ciertos factores ambientales e intrapersonales que pueden influir en la satisfacción de los estudiantes con la escuela. Estas variables incluyen: el comportamiento del profesor de apoyo y ambiente de la clase-escuela, las relaciones positivas con sus compañeros, la calidad de la vida familiar, autoeficacia académica.

Osterman (2000), estudió las variables que en la escuela determinan la satisfacción de las necesidades psicológicas, y cómo las características del contexto social pueden afectar dicha determinación. La preocupación en este estudio fue demostrar cómo las escuelas, siendo organizaciones sociales, hacen frente a las necesidades psicológicas básicas, principalmente la necesidad de experimentar pertenencia. Los resultados sugieren que los estudiantes que experimentan aceptación y valoración, son afectados en las múltiples dimensiones de su comportamiento, por lo que se recomienda que las escuelas adopten prácticas organizativas diligentes para mejorar los medios que valoren el esfuerzo académico y así apoyar a los estudiantes en su experiencia de pertenencia a la comunidad.

Verkuyten y Thijs (2002), observaron la influencia que tiene sobre la valoración de la satisfacción educativa las variables del rendimiento escolar y maltrato, además de la discriminación por las variables de raza y género. Entre sus conclusiones, sugieren que los estudiantes al sentirse académicamente competentes elevan los niveles de satisfacción con la escuela. En este estudio se concluye además, que la satisfacción esta determinada por las características de las relaciones entre pares, además observaron que las capacidades pedagógicas de los maestros, sobre todo en actuaciones positivas ante casos de victimización, variaba considerablemente los niveles de satisfacción. Elmore y Huebner (2010) se enfocaron en las relaciones entre las variables más influyentes que los estudiantes seleccionan para valorar las condiciones del centro educativo. Entre las conclusiones presentadas, sugiere que el disfrute, es un buen indicador para la satisfacción con la escuela. Esto nos lleva a considerar de nuevo que el bienestar subjetivo es un importante factor de la satisfacción.

Galíndez y Casas (2010), advirtieron las relaciones entre la vida familiar y la satisfacción con la escuela. Entre los resultados se sugiere que los adolescentes reportan niveles altos de satisfacción con sus vidas globalmente, y también con sus familias, sus amigos, su entorno y consigo mismos. Sin embargo, estos adolescentes están sólo moderadamente satisfechos con la escuela. Por otro lado, se ha constatado que hay una estrecha relación entre la satisfacción con la vida que muestran los adolescentes y la percepción que tienen de su familia. Concretamente, la satisfacción vital de los adolescentes. 
Zullig, Huebner y Patton (2011), estudiaron la magnitud de las relaciones entre los ocho dominios del clima escolar y una medida de satisfacción escuela global. Entre los resultados del análisis de regresión múltiple sugieren que cinco dominios de clima escolar están significativamente relacionados con la satisfacción escolar: apoyo académico, relaciones positivas docente-estudiante, vínculo con la escuela, orden y disciplina, y la satisfacción académica. Además, la importancia de las variables del clima escolar con la satisfacción escolar de los estudiantes parecía invariable entre las variables demográficas y los niveles de rendimiento académico.

Los anteriores estudios nos permiten considerar la importancia que tiene relacionar los diferentes factores (en nuestro caso componentes) que forman parte de la gestión educativa, para comprender cómo el centro educativo gestiona positivamente el ambiente escolar y establece mecanismos de control interno entre las diferentes relaciones interpersonales (estudiante-estudiante, estudiante-profesor) que hacen posible crear y recrear un clima escolar seguro y confiable. En este trabajo consideramos importante investigar la relación entre los aspectos que hacen parte de clima escolar, para vincularla con otros contextos emocionalmente importantes como son el social y el familiar. Dicha vinculación la llevamos a cabo con el fin de estimar la implicación de la eficacia de la educación en el conjunto de la vida, y para ello el bienestar subjetivo es el mejor indicador de qué también la escuela está respondiendo ante las necesidades formativas y emocionales de sus estudiantes. La gestión educativa puede ampliar y mejorar sus mecanismos de evaluación para lograr una educación de calidad, en tanto perciba la implicación que tienen los diferentes procedimientos programados y planificados en el estudiantado, y para ello, la relación con la vida en general es la mejor manera de conocer sus efectos.

\section{La satisfacción estudiantil como medida de componentes}

Basado en los modelos integrados de Scheerens y Stufflebeam, hemos buscado relacionar los componentes y comprobar su interrelación, estructurando un instrumento de medida que indague acerca de la satisfacción en los estudiantes. Se ha escogido tres instrumentos que están relacionados con los componentes que deseamos estudiar. La selección de estos instrumentos se hace partiendo de los contenidos encontrados en las subdimensiones correspondientes.

El primer componente input, que corresponde a todas aquellas acciones, medios y recursos planificados con los que cuenta el centro para brindar el servicio educativo, nos permite elegir el instrumento SERVQUAL, que indaga sobre cinco subdimensiones del servicio: elementos tangibles, empatía, confiabilidad, capacidad de respuesta, y seguridad. El instrumento satisfacción de los estudiantes universitarios con su educación (SEUE), desarrollado por Gento \& Vivas (2003) cuyas variables están contenidas en dos componentes, procesos y producto, nos permite indagar sobre la satisfacción de los estudiantes sobre las subdimensiones: 
proceso de enseñanza aprendizaje, logros personales, reconocimiento del éxito personal, y autorealización. Y el instrumento que nos sirve de orientador para el componente contexto, es el Multidimensional Students Life Satisfaction Scale (MSLSS), desarrollado por Huebner (1991a) que indaga sobre la satisfacción en tres dimensiones: contexto familiar - satisfacción con la familia; contexto social satisfacción con los amigos; y contexto escolar - satisfacción con la escuela.

\section{Investigación}

La satisfacción educativa en adolescentes es posible comprenderla por medio de las manifestaciones o reportes basados en el bienestar subjetivo. Nuestro objeto de estudio es comprender la relación entre los diferentes contextos en los que se lleva a cabo los procesos educativos (contexto escolar, social y familiar) y la influencia que existe entre sus componentes (inputs, proceso y producto) y en esta relación comprender algunas condiciones del bienestar subjetivo.

\section{Objetivo}

Identificar variables significativas al indagar sobre la satisfacción con la calidad de la educación en estudiantes de $3 .^{\circ}$ y $4 .^{\circ}$ de a ESO, en relación con la satisfacción con la vida.

\section{Metodología}

En este trabajo se aplica un método no experimental, en donde el investigador no posee control directo de las variables. Y de acuerdo con Hernández, Fernández y Baptista (2003) es un diseño transaccional, en el cual se busca encontrar correlación o causas entre dos o más variables en un momento específico.

A diferencia de otros estudios, que analizan la satisfacción con la escuela, empleando el instrumento MSLSS (Huebner, Suldo, \& Valois, 2003; Verkuyten \& Thijs 2002; Elmore \& Huebner 2010; Zullig, Huebner \& Patton, 2011), y lo relacionan con variables que contienen aspectos subjetivos o ambientales, en esta pesquisa lo hemos incluido en el componente contexto, y los relacionaremos con los componentes (inputs, proceso y producto). La principal diferencia que podemos hacer en este estudio, frente a los anteriores, consiste en la inclusión de subdimensiones no vinculadas propiamente con el bienestar subjetivo, como son los elementos tangibles, que hacen hincapié acerca de la valoración con la satisfacción con las instalaciones del centro, los servicios con los que cuenta el aula y el centro; y procedimiento de enseñanza, que hace una cuestión sobre los contenidos del curso, el dominio del docente de la asignatura, el sistema de evaluación, los deberes, las actividades extraescolares, la disponibilidad y uso de las nuevas tecnologías y el asesoramiento de la orientación. De forma similar, se han identificado subdimensiones que están en 
estrecha relación con el bienestar subjetivo, como en las condiciones ambientales positivas: empatía, fiabilidad, seguridad que hacen parte del componente inputs. Las subdimensiones logros personales, reconocimiento del éxito y autorealización, como parte del componente producto.

En el estudio realizado por Zullig, Huebner \& Patton (2011), emplearon el análisis de regresión a fin de conocer los dominios del clima escolar que estaban significativamente relacionados con la satisfacción con la vida. El apoyo académico, las relaciones positivas estudiante docente, los vínculos con la escuela, el orden, la disciplina, y la satisfacción académica, fueron los dominios predictores para la satisfacción con la vida. Este estudio nos sirve de modelo para emplear esta técnica de análisis.

\section{Instrumentos de recogida de datos}

Los instrumentos de medida seleccionados contienen los componentes que se buscan relacionar y se han considerado adecuados para nuestro objetivo de estudio (Gil, 2011), sobre todo, por contener entre sus subdimensiones ítems que cuestionan tanto aspectos del bienestar subjetivo como ambiental. Y la presentación del instrumento a los estudiantes se propone para su valoración en una escala tipo Likert que va desde 1 totalmente en desacuerdo a 7 totalmente en acuerdo.

El instrumento ha pasado por un tratamiento analítico, siguiendo la recomendación de revisión de expertos, además se aplicó una prueba piloto. Para la revisión por los expertos se formularon tres criterios: el primero fue la capacidad comprensiva de los ítems. Cada uno de los ítems fue evaluado en la capacidad de ser comprendido y entendido, sobre todo, considerando que indagan acerca de la satisfacción con la educación. El segundo, se basó en la capacidad resolutiva. La correcta redacción y la adecuada utilización de los términos, buscando no generar ambigüedades al momento de valorar los ítems en la escala propuesta. Y el tercero es relacionado con la capacidad indagativa de la satisfacción educativa. Este aspecto buscó crear una cuestión acerca de la objetividad de los ítems para medir la calidad de la educación por medio de la satisfacción. Se realizó una prueba piloto del instrumento aplicándolo en una muestra con 85 estudiantes, para evaluar las condiciones de validez y fiabilidad con los datos recogidos. Con la finalidad de mejorar la fiabilidad del MSLSS, y lograr una mejor selección factorial de los ítems, se aplicó un análisis factorial hasta lograr una conformación de dos componentes. 
Tabla 1. Ítems MSLSS factorizado y estadísticos de fiabilidad.

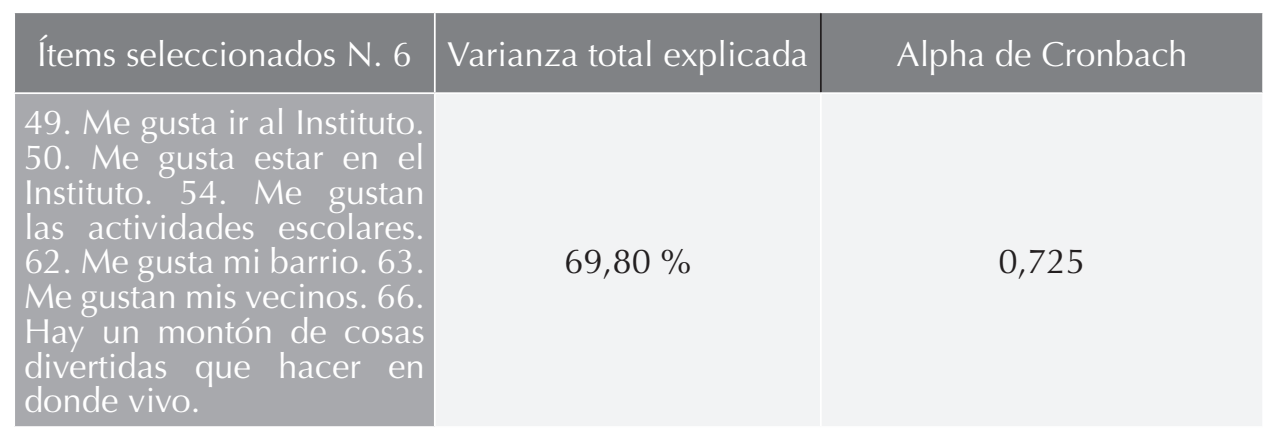

Fuente: Elaboración propia

La composición del instrumento MSLSS que mejor fiabilidad obtuvo, fue aquella conformada por los seis ítems descritos en la tabla 1. Esta composición alcanzaba una varianza explicativa del 69,80\%.

\section{Análisis e interpretación de datos}

El análisis de regresión es una forma de modelar la relación entre una variable dependiente y otras independientes, en un término aleatorio determinado. En nuestro estudio se desea conocer cómo la satisfacción con la escuela (variables independientes), puede ser comprendida de formar específica a partir de la satisfacción con la vida (variable dependiente). Los datos son analizados en el programa SPSS, versión 19, para Windows.

\section{Resultados del estudio}

DLa encuesta de satisfacción educativa fue aplicada a un grupo de 235 estudiantes de secundaria, de $3 .^{\circ}$ y $4 .^{\circ}$ de la ESO.

Tabla 2. Muestra por género.

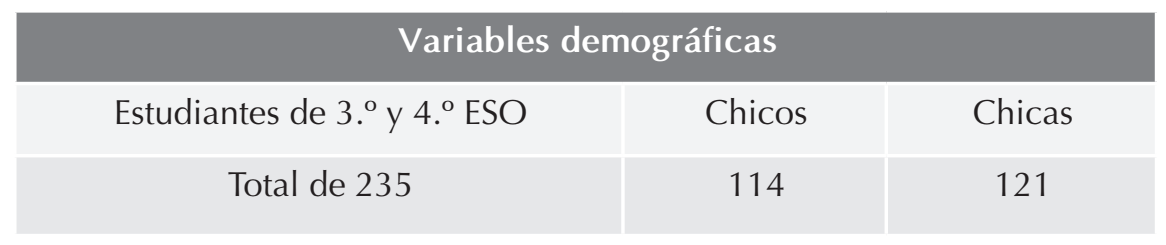

Fuente: Elaboración propia 
Estos datos son tomados en tres centros educativos. Dos de ellos de concertados y uno de titularidad pública.

El análisis estadístico de los datos recogidos, se dividió en dos fases. La primera es el análisis de los estadísticos descriptivos más relevantes, es decir, las medias y la desviación típica. La segunda fase es el estudio de la correlación entre las variables. Para tal fin se realizaron correlaciones con el propósito de conocer si existía asociación positiva significativa entre la satisfacción estudiantil con la vida (componente contexto), y las subdimensiones empatía, confiabilidad, capacidad de respuesta, seguridad, proceso de enseñanza aprendizaje, logros personales, reconocimiento del éxito personal y autorealización. Así mismo, mediante el método de regresión lineal múltiple, se analizó la capacidad predictiva de la satisfacción con la vida y los componentes. Es importante destacar que para realizar estos análisis se han agrupado los ítems en relación con su subdimensión específica. Cada instrumento es analizado en conjunto y por subdimensiones.

\section{Análisis descriptivos}

Los ítems que mejor valoración obtienen en el conjunto de las puntuaciones, están muy relacionados con los aspectos que descubrimos influyentes en el análisis de regresión. Fueron 8 los ítems mejor valorados y que corresponden a la subdimensión, contexto familiar. Las siguientes tablas son los resultados medios de las subdimensiones por instrumento-componente.

Tabla 3. Estadísticos descriptivos SERVQUAL

\begin{tabular}{|c|c|c|c|c|}
\hline & N & Suma & Media & Desv. típ. \\
\hline TANGIBLES & 235 & 991,00 & 4,2170 & 0,91592 \\
\hline EMPATÍA & 235 & 1125,80 & 4,7906 & 0,89313 \\
\hline CAPACIDAD & 235 & 1082,72 & 4,6073 & 0,84395 \\
\hline SEGURIDAD & 235 & 1186,97 & 5,0509 & 1,14088 \\
\hline
\end{tabular}

Tabla 4. Estadísticos descriptivos SEUE

\begin{tabular}{|c|c|c|c|r|}
\hline & N & Suma & Media & Desv. típ. \\
\hline PROC. ENSEÑANZA 1 & 235 & 1190,53 & 5,0661 & 1,00538 \\
\hline PROC. ENSEÑANZA 2 & 235 & 1159,63 & 4,9346 & 1,00490 \\
\hline LOGROS PERSONALES & 235 & 1222,34 & 5,2014 & 0,99371 \\
\hline RECONOCIMIENTO ÉXITO & 235 & 1202,40 & 5,1166 & 1,06964 \\
\hline AUTOREALIZACIÓN & 235 & 1220,31 & 5,1928 & 96842 \\
\hline
\end{tabular}


Tabla 5. Estadísticos descriptivos MSLSS

\begin{tabular}{|c|c|c|c|c|}
\hline CONTEXTO ESCOLAR & N & Suma & Media & Desv. típ. \\
\hline CONTEXTO SOCIOCULTURAL & 235 & 914,79 & 3,8927 & 1,11490 \\
\hline CONTEXTO FAMILIAR & 235 & 1152,07 & 4,9024 & 0,91025 \\
\hline
\end{tabular}

Podemos distinguir las subdimensiones seguridad 5,05; logros Personales 5,20; autorrealización 5,19; y contexto familiar 5,2, como aquellas con mejor reporte de puntuación valorativa. A fin de lograr una mejor fiabilidad de los datos, se ha realizado una reorganización de los ítems. El MSLSS, fue factorizado tomando como referencia la prueba piloto. Este se configuró en dos componentes logrando un alfa de Cronbach de 0,79, y un acumulado de varianza explicada del $64,8 \%$.

\section{Resultados del análisis de regresión}

Se inicia el análisis en la selección de los mejores datos de significación estadística entre los diferentes componentes y las subdimensiones. Se ha seleccionado el método de análisis introducir por ser considerado uno de los más adecuados para la selección de variables que mayor peso puedan obtener (Gil, 2011). Las siguientes tablas recogen los resultados logrados:

Tabla 6. Coeficientes ${ }^{a}$ INSTRUMENTOS

\begin{tabular}{|c|c|c|c|c|c|c|c|}
\hline \multirow[t]{2}{*}{ Modelo } & \multirow[t]{2}{*}{ Coeficiente: } & \multicolumn{2}{|c|}{ s no estandarizados } & \multicolumn{3}{|c|}{ Coeficientes tipificados } & \multirow[t]{2}{*}{ Sig. } \\
\hline & & B & Error tip & & Beta & & \\
\hline 1 & (Constante) & 1,678 & & 0,399 & & 4,211 & 0,000 \\
\hline & SEUE2 & 0,680 & & 0,090 & 0,598 & 7,572 & 0,000 \\
\hline & SEUE1 & 0,025 & & 0,109 & 0,023 & 0,232 & 0,816 \\
\hline & SERVQUAL & $-0,091$ & & 0,123 & $-0,061$ & $-0,742$ & 0,459 \\
\hline
\end{tabular}

Fuente: Elaboración propia

a. Variable dependiente: MSLSSfactor

Se observó que el componente que resulta más significativo es SEUE2, por lo que se considera la necesidad de analizar otros modelos, por instrumento, a fin de comprobar la significación por separado. Realizando estos análisis se obtiene que las subdimensiones de los coeficientes de significación, siguen estando relacionados con el componente input y el componente producto. 
Tabla 7. Coeficientes ${ }^{a}$ con mejor significación

\begin{tabular}{|lccccc}
\hline $\begin{array}{c}\text { Coeficientes no } \\
\text { estandarizados }\end{array}$ & B & \multicolumn{2}{c}{$\begin{array}{c}\text { Coeficientes } \\
\text { tipificados }\end{array}$} & t & Sig. \\
\hline EMPATÍA & 0,435 & 0,091 & 0,034 & 4,768 & 0,000 \\
\hline LOGROS PERSONALES & 0,299 & 0,089 & 0,286 & 3,363 & 0,001 \\
\hline RECONOCIMIENTO ÉXITO & 0,259 & 0,074 & 0,266 & 3,504 & 0,001 \\
\hline
\end{tabular}

Fuente: Elaboración propia

a. Variable dependiente: MSLSSfactor

Las subdimensiones empatía, logros personales y reconocimiento del éxito, son las que resultan significativas y que poseen una mejor determinación relacional y para comprobar que dicha significación es relevante, se han realizado los supuestos necesarios para conocer mejor las características. La revisión del primer supuesto por medio de la nube de puntos, donde los puntos residuales nos ofrecen una visualización general de la regresión, nos permitió observar que se puede lograr un aumento de la satisfacción con la vida basados en los aspectos relativos a la empatía, los logros personales, como del reconocimiento del éxito personal. A pesar de que se observó una gran dispersión, gran parte de los puntos de la nube se encontraron entre los lineales 4 y 6 . Además, se evalúo el diagrama por medio del R2: empatía 0,15; logros personales 0,26; y reconocimiento del éxito, 0,25. Estos datos nos permiten observar que son muchas las puntuaciones dispersas, pero otros están relativamente centrados en este rango.

La fórmula de la recta, nos muestra que el logro personal obtiene la mejor relación en la recta lineal con 0,26. Las pendientes de las rectas indican que, en promedio, a cada incremento de valoración en la satisfacción con las diferentes subdimensiones (contexto escolar, familiar y social), le corresponde un incremento en las subdimensiones empatía, logros personales y reconocimiento del éxito. Siguiendo con los supuestos requeridos para el análisis de regresión, se analizó la bondad de ajuste. Las tres variables independientes incluidas en el análisis explican un $33 \%$ de la varianza de la variable dependiente, pues $\mathrm{R}^{2}$ corregida $=$ 0,32 . Además, el error típico de los residuos es 0,85559 en el análisis de regresión múltiple, lo que indica una pequeña mejora en el ajuste. El valor corregido de $\mathrm{R}^{2}$ es casi idéntico al valor no corregido.

El estadístico $F$, permite evidenciar la relación dependiente de las variable significativa en la covarianza, encontrando el valor poblacional de $\boldsymbol{R}$ es cero y, por tanto, nos permite decidir si existe relación lineal significativa entre la variable dependiente y el conjunto de variables independientes tomadas a la vez. El valor del nivel crítico sig. $=0,000$ indicando que si existe relación lineal significativa. Podemos afirmar, por tanto, que el hiperplano definido por la ecuación de regresión ofrece 
un buen ajuste a la nube de puntos. En la tabla del ANOVA se observó la relación significativa entre las variables. El nivel crítico (sig.) indica que el valor poblacional de $\mathrm{R}$, en esta muestra, tome el valor 0,575. Lo que implica que $\mathrm{R}$ fue mayor que cero y que, en consecuencia, las variables están linealmente relacionadas, aspectos que nos permite afirmar que la significación que los estudiantes encuentren con la calidad de la educación estará relacionada con la calidad de vida en sus otros contextos.

Otro supuesto necesario es la ecuación de regresión. El pronóstico de relación en la satisfacción fue igual a 1,304. Estos coeficientes no estandarizados podemos interpretarlos en los siguientes términos: el coeficiente correspondiente a la variable logros personales, que vale 0,287 , indica que si el resto de variables se mantienen constantes, a un aumento a la valoración de la satisfacción con la educación, corresponderá un aumento de satisfacción con la vida (MSLSSfactor). Es necesario señalar que estos coeficientes no son independientes entre sí. De hecho, reciben el nombre de coeficientes de regresión parcial porque el valor concreto estimado para cada coeficiente se ajusta teniendo en cuenta la presencia del resto de variables independientes, y acercándose a una interpretación de primer orden es posible que logros personales son los puntos focales de consideración para los estudiantes cuando asignan valor estimativo a la calidad de la educación que reciben.

\section{Conclusiones}

En este estudio se investigó la relación explicativa que puede tener la valoración de la calidad de la educación por medio de la satisfacción, en la satisfacción con la vida discriminada en los contextos educativos familiar, social cultural y escolar, evaluando las características de dicha relación. En general, los estudiantes valoran positivamente las diferentes subdimensiones. Por otra parte, los ítems mejor valorados, superan 5,30 y están relacionados con las dimensiones que fueron influyentes en el modelo de regresión propuesto.

Las subdimensiones, variables independientes, que resultaron más significativas en el modelo de regresión empatía, logros personales y reconocimiento del éxito, nos ayudan a comprender cómo influyen sobre la satisfacción de los estudiantes, la educación que reciben basados en la satisfacción con la vida. La variable, logros personales, que corresponde a los diversos incentivos institucionales y personales con relación a la valoración del esfuerzo particular, fue la subdimensión más significativa del modelo propuesto. Estos aspectos nos permiten aceptar que la medida de la calidad de la educación valorada por medio de la satisfacción y fundamentada en el bienestar subjetivo, tiene una relación determinante sobre la 
valoración en general de la satisfacción con la vida, puesto que esta valoración se fundamenta en las condiciones socias emocionales que hacen parte del conjunto de acciones educativas en el contexto escolar. La valoración de las capacidades, como forma del reconocimiento del esfuerzo, también observado por McCullough, Huebner y Laughlin (2000), sugiriere que los estudiantes con mayor autoeficacia académica suelen reportar mejores niveles de satisfacción con la escuela. De forma similar (Zullig \& Huebner 2011), quienes concluyeron en su estudio, que los dominios del clima escolar que más influyen en la satisfacción corresponden a aquellos relacionados con el fortalecimiento del bienestar subjetivo, en nuestro estudio se han distinguido tres subdimensiones que también están muy relacionadas con este concepto. Las subdimensiones que fueron relacionadas, corresponden al componente input (empatía), y el componente producto (logros personales y reconocimiento del éxito), quedando por fuera del análisis subdimensiones del componente proceso. Este aspecto nos lleva a considerar las siguientes ideas:

En la investigación Ilevada a cabo por Verkuyten y Thijs (2002), se concluye que la actuación docente puede ser determinante para la satisfacción con la educación, en tanto que los acontecimientos que modificaban esta determinación estaban relacionados con la victimización. En nuestro estudio, los ítems que indagan sobre la actuación docente, contenidos en el componente Proceso: dominio docente, exigencia, sistema de evaluación, deberes, actividades extraescolares, asistencia docente, nuevas tecnologías y orientación, no representaron en el modelo propuesto significación alguna. Esto nos hace preguntarnos sobre la determinación que pueden tener para la valoración de la satisfacción los aspectos metodológicos. Por lo que comparando nuestro estudio con Verkuyten y Thijs, podemos Ilegar a formular que la implicación pedagógica de la actuación docente puede ser medida desde otras dimensiones, como pueden ser los aportes emocionales que ofrece la actuación docente para la construcción de la personalidad.

En relación con la subdimensión elementos tangibles, que indaga sobre las instalaciones del centro, sobre los servicios del aula, entre otros, el modelo de regresión no reportó relación significativa. En el estudio realizado por Zullig y Huebner y Patton (2011), citando a Ho y Smith (2006), concluyen que los aspectos relacionados con las instalaciones del centro, o los suministros, no reportan ser influyentes en las variables que determinan la satisfacción de los estudiantes. Esta comparación nos permite considerar sobre cuál es la mejor forma de evaluar la repercusión que tiene sobre la satisfacción las condiciones ambientales locativas.

Las subdimensiones confianza, capacidad y seguridad, tampoco resultaron significativas. En los trabajos realizados por Huebner (1994) y Huebner, Suldo, y Valois (2003), se concluye para la satisfacción con la escuela son más influyentes las variables vinculadas con las relaciones entre sus familiares y compañeros, antes que las condiciones relacionadas con el clima escolar fuera del aula. Estos aspectos 
nos permiten acentuar que existen variables más influyentes para los estudiantes. La integridad y la seguridad emocional que parte de las relaciones establecidas entre compañeros, puede crear una base para considerar la calidad de la educación.

La subdimensión, autorealización, que contiene aspectos relacionados con la importancia que tiene la educación para la vida, la adquisición de conocimientos y destrezas, el fortalecimiento de la autonomía, y el desarrollo de la creatividad, tampoco reportó una relación significativa para el modelo propuesto. Frente a este resultado, resulta muy contradictorio nuestro estudio al compararlos con otros estudios. Huebner, (1991) y Alderman y Huebner, (1993), encontraron que la autoestima tiene un efecto directo sobre la satisfacción con la escuela.

Este estudio sugiere que la medida de la satisfacción de los estudiantes con la experiencia educativa no puede ser considerada únicamente como un producto de la calidad de la acción educativa, puesto que este puede ser comprendido desde una relación de componentes y en dicha relación existen variables que son más influyentes que otras. Las variables que se han encontrado más significativas, pueden ayudarnos a superar una visión reducida de la satisfacción, enfatizando áreas de la gestión del centro que pueden ser igualmente fundamentadas y que los estudiantes estiman mejor. El modelo de regresión lineal, nos evidencia que la significación es determinante de la estimación de la calidad.

La principal idea que podemos considerar, es que la satisfacción de los estudiantes con la calidad de la educación percibida, está muy relacionada con las condiciones socioemocionales, las valoraciones afectivas del esfuerzo y el reconocimiento de logros. Las expectativas con la educación, evaluadas desde el conjunto de la vida, son un camino oportuno para la valoración de la calidad de la acción educativa en una continua relación de variables. El reconocimiento de la relación de dichas variables, como la determinación entre ellas puede aportar información necesaria para el mejoramiento de la práctica docente.

Consideramos que el modelo de regresión hallado, posee limitaciones. En primer lugar, los datos se obtuvieron en tres centros educativos, dos concertados y uno de titularidad pública, de los estudiantes de una población semiurbana, con lo que la generalización de los hallazgos está restringido únicamente a estos, y se necesita más investigación para conocer si estos resultados se aplican a otras poblaciones de estudiantes. En segundo lugar, este estudio de corte transversal no tiene en cuenta aspectos influyentes a la hora de cuestionar acerca de la valoración que hacen los estudiantes sobre la calidad de la educación que reciben (exámenes previos, situaciones grupales específicas, etc.).

Un estudio adicional puede llevarse a cabo en diferentes poblaciones, para identificar si las variables relacionadas significativamente consisten en otros grupos 
de estudiantes. Además, ampliar y comparar con variables demográficas puede ser significativo en el mismo modelo de regresión. Luego, el MSLSS es un instrumento que ayuda a comprender la relación determinante de la satisfacción en la época escolar, pero en este estudio se redujo por la factorización, hecho que nos lleva a plantear la necesidad de volver a revisar esta configuración para posteriores estudios. 


\section{Referencias}

Antaramian, S., Huebner, E., \& Valois, R. (2008). Adolescent Life Satisfaction. Applied psychology: An international review (57), 112-126.

Baker, J. (1998). The social context of school satisfation among urban low in come, AfricaAmerican student. School Psychilogy Quartely, 25-44.

Baker, J. A., Dilly, L. J., Aupperlee, J., \& Patil, S. (2003). The developmental context of school satisfaction: School as psychologically healthy environments. School Psychology Quarterly(18), 206-221.

Diener, E. (1994). Assessing subjective well-being: Progress and opportunities. Social Indicators Research(31), 103-159.

Diener, E., Suh, E., Lucas, R., \& Smith, H. (1999). Subjective well-being: three decades of progress. Psychol. Bul/(125), 276-302.

Elmore, G., \& Huebner, E. (2010). Adolescent's satisfaction with school experiences: Relationships with demographics, attachment relationships, and school engagement behavior. Psychology in the Schools, 47(6), 37-79.

Elmore, G., \& Huebner, E.S. (2010). Adolescent's satisfaction with school experiences: Relationships with demographics, attachment relationships, and school engagement behavior. Psychology in the Schools, 47(6), 80-107.

Epstein, J., \& McPartland, J. (1976). The concept and measurement of the quality of school life. American Educational Research Journal(13), 15-30.

Galán, A. (2004). Evaluación del clima escolar como factor de calidad. Madrid, España: Muralla.

Galindez, E., \& Casas, F. (2010). Adaptación y validación de la Students' Life Satisfaction Scale (SLSS) con adolescentes. Estudios de Psicología(31 (1)), 79-87.

Gardner, H. (1993). La mente no escolarizada. Barcelana: Paidós.

Gardner, H. (1999). Inteligencias Múltiples. La teoría en la práctica. Barcelona: Paidos.

Gento, S. (2000). Organización y gestión de instituciones educativas. IX(Unidad 52), 42.

Gento, S., \& Vivas, M. (2003). El SEUE: un instrumento para conocer la satisfacción de los estudiantes universitarios con su educación. Acción Pedagógica, 12(2), 16-27.

Gil, J. A. (2011). Técnicas e instrumentos para la recogida de información. Madrid: España: UNED.

Hernández, S. R., Fernández, C., \& Baptista, L.P. (2003). Metodología de la investigación. México: McGraw Hill.

Huebner, E. (1991a). Initial development of the Students' Life Satisfaction Scale. School Psychology International(12), 231-240.

Huebner, E. (1991b). Correlates of life satisfaction in children. School Psychology Quarterly(6), 103-111.

Huebner, E. S. (1994). Preliminary development and validation of a multidimensional life satisfaction scale for children. Psychological Assessment(6), 149-158. 
Huebner, E. S. (2004). Research on assessment of life satisfaction in children and adolescents. Social Indicators Research(66), 3-33.

Huebner, E. S., Suldo, S., \& Valois, R. (2003). Psychometric Properties of Two Brief Measures of Children's Life Satisfaction: The Students' Life Satisfaction Scale (SLSS) and the Brief Multidimensional Students' Life Satisfaction Scale (BMSLSS)., (págs. March 12-13). California.

Huebner, E. S., Valois, R., Paxton, R., \& Drane, J. (2005). Middle school student's perceptions of quality of life. Journal of Happiness Studies(6), 15-24.

McCullough, G., Huebner, S, \& Laughlin, J. (2000). Life events, self-concept, and adolescent's positive subjective well-being. Psychology in the Schools, 37(3), 201-301.

Morin, E. (2002a). Educar en la era planetaria. España: Gedisa.

Osterman, K. (2000). Students' Need for Belonging in the School Community. Review of Educational Research Fall(70), 323-367.

Peréz, R. (2005). La calidad de la educación, la educación con calidad. Hacia su necesaria integración. Revista Educación XX1, 8, 11-33.

Pérez, R. (2006). Evaluación de programas educativos. Madrid, España: Muralla.

Pérez, R., Galán, A., \& Quintanal, J. (2012). Métodos y diseños de investigación en educación. Madrid, España: UNED Educación.

Seligman, M., \& Csikszentmihalyi, M. (2000). Positive Psychology: An Introduction. American Psychologist(1), 5-14.

Verkuyten, M., \& Thijs, J. (2002). School satisfaction of elementary school children: the role of performance, peer relations, ethnicity and gender. Social Indicators Research(59), 203228.

Zullig, K., Huebner, E., \& Patton, J. (2011). Relationships among school climate domains and school satisfaction. Psychology in the Schools(48 (2)), 78-97.

Recibido: 1 de octubre 2014

Aceptado: 21 diciembre 2014

Cómo citar:

Ríos, A. (2015). Medida de la satisfacción estudiantil a partir de la satisfacción con la vida: una relación de componentes. Praxis Pedagógica, 16, 17-37. 